\title{
Practical Performances of Non-linear Algorithms for Partitioned Iterative Method of Fluid-Structure Interaction Problems*
}

\author{
Satsuki MINAMI** and Shinobu YOSHIMURA ${ }^{* * *}$ \\ ** University of Tokyo, Department of Quantum Engineering and Systems Science \\ 7-3-1 Hongo, Bunkyo-ku, Tokyo 113-8656, Japan \\ E-mail: minami@save.sys.u-tokyo.ac.jp \\ *** University of Tokyo, Department of Systems Innovation
}

\begin{abstract}
Recently, tightly coupled partitioned iterative methods have drawn a great deal of attentions due to easy implementation and encapsulation features, and several nonlinear algorithms have been proposed so far. However, their practical performances have not been well understood yet. This paper describes the intensive parametric study on convergence and stability performances of four nonlinear algorithms and their relaxed variations for partitioned iterative methods of steady / unsteady fluid-structure interaction (FSI) problems. Here we choose three typical FSI problems as test problems, i.e. (1) Collapsible channel as a steady problem, (2) Cavity with flexible bottom membrane and (3) Channel with flexible wall as unsteady problems. Efficiency and robustness dependency of those nonlinear algorithms on physical parameters such as degree of nonlinearity, added mass effect, time step, and on control parameters peculiar to each algorithm are clarified. Through those tests, we demonstrate that Broyden method is the fastest algorithm for easy FSI problems such as weakly coupling and Line Search method has robustness even for difficult FSI problems such as strongly coupling.
\end{abstract}

Key words : Partitioned Iterative Method, Non-linear Algorithms, Fluid-Structure Interaction Problems

\section{1. introduction}

Fluid-structure interaction (FSI) problems arise from the phenomena where fluid and structure interact with each other, and recently great importance on those matters is pointed out in various engineering fields. Numerical methods developed for FSI problems can be classified into monolithic and partitioned approaches. The monolithic approaches ${ }^{(1),(2)}$ are generally acknowledged to be accurate and robust, and can be applied for solving problems with strong interaction. On the other hand, the partitioned approaches ${ }^{(3)-(11)}$, which solve fluid and structure problems separately, have been widely studied due to easy implementation and encapsulation features. It is expected that the partitioned approaches seem suitable to construct a large scale parallel FSI analysis system by employing existing parallel codes such as ADVENTURE ${ }^{(12),(13)}$ and FrontFlow ${ }^{(14)}$.

Nowadays, partitioned iterative methods, which improve accuracy and robustness of the partitioned approaches, have attracted a great deal of attention. In the partitioned iterative methods, a fluid problem and a structure problem are solved separately and iteratively until interface conditions of equilibrium and geometrical compatibility are satisfied in each time step. It should be noted that a simple staggered scheme solves the fluid and structure problems only once. Several algorithms such as Block Gauss-Seidel methods ${ }^{(4)}$, Line Search method ${ }^{(5)}$ and Newton-Krylov methods ${ }^{(6)}$ have been proposed as partitioned iterative methods. Although each researcher enhances good points of his / her proposing method, it is known that physical 
parameters such as added mass effect, nonlinearity, time step, degree of incompressibility, selection of time integration scheme, and control parameters of each method affect convergence property of those iterative methods to great extent. Because of such features, it is difficult for an ordinary engineer to select a nonlinear algorithm appropriate for his / her own FSI problem.

In this respect, it is getting important to investigate practical performances of each nonlinear algorithm for various FSI problems. Matthies et al. ${ }^{(7)}$ describe a framework of algorithms for tightly coupled procedures and compare the performances of several nonlinear algorithms. However, this research focuses on general strongly coupling problems and the investigation of performance dependency on the physical parameters of FSI problems is not sufficiently carried out. Kuttler and Wall ${ }^{(8)}$ compared some partitioned iterative methods by changing the amount of added mass effect. However, in this research, a study of comparison was restricted to fixed-point methods with dynamic relaxation.

Nonlinear algorithms can be classified into two kinds: the method using only values of functions, and the one using values of functions as well as information of Jacobi matrices. Gauss-Seidel method, Line Search method, Quasi-Newton method are members of the former method, while Exact Newton method and Inexact Newton method are those of the latter method. There are many variations in each method. The present authors have been developing a general-purpose large-scale FSI analysis system integrating a parallel solid solver and a parallel fluid one by partitioned iterative approaches. For such research, we need to select nonlinear algorithms, which meet the following three requirements: (1) an algorithm does not need to calculate Jacobi matrices explicitly, (2) an algorithm is easy to implement, i.e. additional programming into solid and fluid solvers is less, and (3) an algorithm uses less additional memory due to FSI analysis.

Thus in this paper we perform the intensive parametric study on convergence and stability performances of four nonlinear algorithms and their relaxed variations for partitioned iterative methods of steady / unsteady FSI problems. Here we examine performance dependency of nonlinear algorithms on degree of nonlinearity and initial values for a steady problem, and those on added mass effect and time step size for unsteady problems, and those on control parameters peculiar to each algorithm for both steady / unsteady problems.

The reminder of this paper is organized as follows: In $\S 2$, the governing equations of FSI problems and the overview of partitioned iterative methods are presented. Four nonlinear algorithms and their relaxed variations to be compared are briefly explained in $\$ 3$. Three test FSI problems are introduced and the results of numerical tests are presented in $\S 4$. Finally the conclusion is given in $\S 5$.

\section{Governing Equations of FSI Problems}

\subsection{Fluid Domain}

A fluid domain is governed by the following Navier-Stokes equation in an ALE frame of reference and the equation of continuity:

$$
\begin{aligned}
& \rho^{F} \frac{\partial \mathbf{u}}{\partial t}+\rho^{F}(\mathbf{u}-\hat{\mathbf{u}}) \cdot \nabla \mathbf{u}-\frac{\partial \sigma^{\mathbf{F}}}{\partial \mathbf{x}}=\mathbf{f}, \\
& \nabla \cdot \mathbf{u}=0,
\end{aligned}
$$

where $\rho^{F}$ denotes the fluid density, $\mathbf{u}$ the fluid velocity, $\hat{\mathbf{u}}$ the mesh velocity, $\sigma^{\mathbf{F}}$ the stress tensor of the fluid, and $\mathbf{f}$ the body force applied on the fluid. A stabilized finite element method with first order triangular elements is used for spatial discretization, and the backward Euler scheme for time integration.

\subsection{Structure Domain}

A structure domain is governed by the following equation:

$$
\rho^{S} \frac{\partial^{2} \mathbf{d}}{\partial t^{2}}-\frac{\partial \sigma^{\mathbf{S}}}{\partial \mathbf{x}}=\rho^{S} \mathbf{g}
$$


where $\rho^{S}$ denotes the structural density, $\mathbf{d}$ the structural displacement, $\sigma^{\mathbf{S}}$ the stress tensor of the structure, $\mathbf{g}$ the body force applied on the structure. In this work, a linear St.-VenantKirchhoff material is employed. The Total Lagrangian method with second order quadrilateral elements is used for spatial discretisation, and Newmark- $\beta$ scheme for time integration.

\subsection{Interface between Fluid and Structure Domains}

At the FSI interface, the following conditions of equilibrium and geometrical compatibility are required:

$$
\begin{aligned}
& \mathbf{d}_{\boldsymbol{\Gamma}}=\mathbf{p}_{\boldsymbol{\Gamma}}, \\
& \sigma_{\boldsymbol{\Gamma}}^{\mathbf{S}} \cdot \mathbf{n}^{\mathbf{S}}=\sigma_{\boldsymbol{\Gamma}}^{\mathbf{F}} \cdot \mathbf{n}^{\mathbf{F}} .
\end{aligned}
$$

where superscripts $S, F$ represents quantity of either structure or fluid, respectively, and subscript $\Gamma$ does quantity at the interface. $\mathbf{p}_{\boldsymbol{\Gamma}}$ denotes the displacement of the fluid interface, $\mathbf{n}$ the normal unit vector at the interface, and $\sigma$ the stress tensor.

\subsection{Mesh Control}

For the fluid domain to follow the movement of the structure, the following mesh control techniques are used: mesh smoothing based on Pseudo-elastic technique ${ }^{(15)}$ for small deformation, and remeshing ${ }^{(16)}$ when mesh quality deteriorates due to large deformation.

\subsection{Re-definition of FSI Problem and Partitioned Iterative Methods}

In the FSI problems, the governing equations of fluid, structure and mesh control need to satisfy the conditions of equilibrium and geometrical compatibility on the interface between fluid and structure domains. Especially, partitioned iterative methods separate the original FSI problem into three components of fluid analysis $F$, structure analysis $S$ and mesh control $M$, and each component is treated as a nonlinear problem in the following:

$$
\begin{aligned}
& \left(\mathbf{X}_{\Gamma}^{\mathbf{k}+\mathbf{1}}\right)=M\left(\mathbf{d}_{\Gamma}^{\mathbf{k}}\right), \\
& \left(\mathbf{u}_{\Gamma}^{\mathbf{k}+\mathbf{1}}, \mathbf{p}_{\Gamma}^{\mathbf{k}+\mathbf{1}}\right)=F\left(\mathbf{X}_{\Gamma}^{\mathbf{k}+\mathbf{1}}\right), \\
& \left(\mathbf{d}_{\Gamma}^{\mathbf{k}+\mathbf{1}}\right)=S\left(\mathbf{u}_{\Gamma}^{\mathbf{k}+\mathbf{1}}, \mathbf{p}_{\Gamma}^{\mathbf{k}+\mathbf{1}}\right),
\end{aligned}
$$

where $\mathbf{d}_{\boldsymbol{\Gamma}}$ denotes the structural displacement, $\mathbf{u}_{\boldsymbol{\Gamma}}$ the velocity of fluid, $\mathbf{p}_{\boldsymbol{\Gamma}}$ the fluid pressure, and $\mathbf{X}_{\Gamma}$ the mesh coordinates on the interface. In the partitioned iterative methods, those equations are solved sequentially and iteratively, and the nonlinear problems due to an advective term in the fluid analysis and a geometrical nonlinear behavior in the structural analysis are handled on each system separately. Superscript $k$ denotes $k$-th iteration. Combining Eqs. (6)-(8), the FSI equation $Z$ can be rewritten as:

$$
\mathbf{d}_{\boldsymbol{\Gamma}}=Z\left(\mathbf{d}_{\boldsymbol{\Gamma}}\right)=S\left(F\left(M\left(\mathbf{d}_{\boldsymbol{\Gamma}}\right)\right)\right)
$$

The residual of the above nonlinear equation is defined as:

$$
R\left(\mathbf{d}_{\mathbf{\Gamma}}\right) \equiv \mathbf{d}_{\mathbf{\Gamma}}-S\left(F\left(M\left(\mathbf{d}_{\mathbf{\Gamma}}\right)\right)\right)
$$

Thus, the FSI analysis can be regarded as the process seeking the structural displacement $\mathbf{d}_{\boldsymbol{\Gamma}}$ on the interface such that

$$
R\left(\mathbf{d}_{\Gamma}\right)=0 .
$$

\section{Nonlinear Coupling Algorithms}

Various nonlinear algorithms could be applied to solve the FSI equation (11) derived in $\S 2$. Among them, based on the points described in the latter half of $\S 1$, i.e. (1) an algorithm does not need to calculate Jacobi matrices explicitly, (2) an algorithm is easy to implement, i.e. additional programming into solid and fluid solvers is less, and (3) an algorithm uses less 
additional memory due to FSI analysis, we select the following four nonlinear algorithms and their relaxed variations, and compare their practical performances in efficiency and robustness.

- Block Gauss-Seidel method and its variation with relaxation

- Line Search method

- Jacobian-Free Newton-Krylov method and its variation with relaxation

- Broyden method and its variation with relaxation

Each algorithm is explained briefly next.

\subsection{Block Gauss-Seidel method and Its Variation with Relaxation}

In Block Gauss-Seidel method ${ }^{(4)}$, physical quantities (i.e. displacement, velocity, pressure) obtained from either fluid or structure solver are simply substituted to another, and the calculation advances successively. The algorithm is given as follows:

Block Gauss-Seidel method:

Step 0: Give an initial assumption $\mathbf{d}_{\mathbf{\Gamma}}^{\mathbf{0}}$. Set $k=0$.

Step 1: Calculate $\mathbf{r}^{\mathbf{k}}=\mathbf{d}_{\Gamma}^{\mathbf{k}}-S\left(F\left(M\left(\mathbf{d}_{\Gamma}^{\mathbf{k}}\right)\right)\right)$.

Step 2: If converged, exit with the solution $\mathbf{d}_{\Gamma}^{\mathbf{k}}$. If not, go to Step 3.

Step 3: $\quad$ Set $\mathbf{d}_{\Gamma}^{\mathbf{k}+\mathbf{1}}=\mathbf{d}_{\boldsymbol{\Gamma}}^{\mathbf{k}}-\mathbf{r}^{\mathbf{k}}$.

Step 4: Set $k=k+1$ : go to Step 1 .

Furthermore, relaxation techniques, which relax the structural displacement $\mathbf{d}_{\boldsymbol{\Gamma}}$ in Step 3 by the following equation, are often introduced in order to stabilize the nonlinear analysis, i.e.

$$
\mathbf{d}_{\Gamma}^{\mathbf{k}+\mathbf{1}}=\mathbf{d}_{\boldsymbol{\Gamma}}^{\mathbf{k}}-\omega^{k} \mathbf{r}^{\mathbf{k}}
$$

There are several variations depending on the selection of the relaxation parameter $\omega^{k}$. The under-relaxation method uses a constant number $\left(0<\omega^{k}<1\right)$. In Aitken extrapolation ${ }^{(9)}$, $\omega^{k}$ is defined in the following:

$$
\omega^{k}=\arg \min \left\|\left(\mathbf{d}_{\Gamma}^{\mathbf{k}}-\mathbf{d}_{\Gamma}^{\mathbf{k}-\mathbf{1}}\right)-\omega^{k}\left(\mathbf{r}^{\mathbf{k}}-\mathbf{r}^{\mathbf{k}-\mathbf{1}}\right)\right\|,
$$

where some appropriate value is chosen as the initial value of $\omega^{0}$. It is comprehensible that Aitken extrapolation is an extension of one-dimensional secant method into vector version, and the parameter $\omega^{k}$ is decided so that the norm of difference between the last two nonequilibrated displacements is minimized.

\subsection{Line Search method}

The algorithm of Line Search method proposed by one of the present authors ${ }^{(5)}$ is given as follows:

\section{Line Search method:}

Step 0: Give an initial value $\mathbf{d}_{\mathbf{\Gamma}}^{\mathbf{0}}, \alpha^{0}$. Set $k=0$.

Step 1: Calculate $\mathbf{r}^{\mathbf{k}}=\mathbf{d}_{\Gamma}^{\mathbf{k}}-S\left(F\left(M\left(\mathbf{d}_{\Gamma}^{\mathbf{k}}\right)\right)\right)$.

Step 2: If converged, exit with the solution $d_{\Gamma}^{k}$. If not, go to Step 3 .

Step 3: Set $\alpha^{k}=\arg \min \left\|\alpha^{k} \mathbf{r}^{\mathbf{k}}+\left(1-\alpha^{k}\right) \mathbf{r}^{\mathbf{k}-\mathbf{1}}\right\|$

Step 4: $\quad \operatorname{Set} \mathbf{d}_{\boldsymbol{\Gamma}}^{\mathbf{k}+\mathbf{1}}=\alpha^{k} \mathbf{d}_{\boldsymbol{\Gamma}}^{\mathbf{k}}+\left(1-\alpha^{k}\right) \mathbf{d}_{\boldsymbol{\Gamma}}^{\mathbf{k}-\mathbf{1}}-\beta\left(\alpha^{k} \mathbf{r}^{\mathbf{k}}+\left(1-\alpha^{k}\right) \mathbf{r}^{\mathbf{k}-\mathbf{1}}\right)$.

Step 5: $\quad$ Set $k=k+1$ : go to Step 1 .

It should be noted that $\alpha^{k}$ is decided so that the norm of linearly interpolated residual is minimized. $\beta(0<\beta \leq 1)$ must be given by a user. $\beta$ is introduced to avoid the situation such that a search space is only on a single line ${ }^{(5)}$.

\subsection{Jacobian-Free Newton-Krylov method and Its Variation with Relaxation}

Newton-Krylov method is a family of Newton method, and uses Krylov Subspace methods for finding a Newton step. The algorithm is as follows: 
Newton-Krylov method:

Step 0: Give an initial assumption $\mathbf{d}_{\Gamma}^{\mathbf{0}}$. set $k=0$.

Step 1: Calculate $\mathbf{r}^{\mathbf{k}}=\mathbf{d}_{\Gamma}^{\mathbf{k}}-S\left(F\left(M\left(\mathbf{d}_{\Gamma}^{\mathbf{k}}\right)\right)\right)$.

Step 2: If converged, exit with the solution $\mathbf{d}_{\Gamma}^{\mathbf{k}}$. If not, go to Step 3 .

Step 3: Find an increment $\mathbf{s}^{\mathbf{k}}$ by solving equation $\mathbf{J}^{\mathbf{k}} \mathbf{s}^{\mathbf{k}}=-\mathbf{r}^{\mathbf{k}}$ with Krylov subspace method.

Step 4: $\quad$ Set $\mathbf{d}_{\boldsymbol{\Gamma}}^{\mathbf{k}+\mathbf{1}}=\mathbf{d}_{\boldsymbol{\Gamma}}^{\mathbf{k}}+\omega^{k} \mathbf{s}^{\mathbf{k}}$.

Step 5: $\quad$ Set $k=k+1$ : go to Step 1 .

In case that Jacobian is not calculated explicitly in Step 3, the algorithm is specifically called Jacobian-Free Newton-Krylov method ${ }^{(17),(18)}$. The following approximate equation is used for calculating the product of Jacobian and a certain vector.

$$
\mathbf{J}^{\mathbf{k}} \mathbf{v} \approx\left[Z\left(\mathbf{d}_{\Gamma}^{\mathbf{k}}+\epsilon \mathbf{v}\right)-Z\left(\mathbf{d}_{\Gamma}^{\mathbf{k}}\right)\right] / \epsilon,
$$

where, $\epsilon$ is a parameter and calculated using a small constant of $\lambda^{(18)}$ by the following:

$$
\epsilon=\lambda \cdot\left(\lambda+\left\|\mathbf{d}_{\mathbf{r}}^{\mathbf{k}}\right\| /\|\mathbf{v}\|\right)
$$

The stop criterion for Krylov subspace method is given as :

$$
\left\|\mathbf{J}^{\mathbf{k}} \mathbf{s}^{\mathbf{k}}-\left(-\mathbf{r}^{\mathbf{k}}\right)\right\|<\epsilon_{0}\left\|-\mathbf{r}^{\mathbf{k}}\right\| .
$$

Here, $\epsilon_{0}$ is a user-defined parameter. Furthermore, $\omega_{k}$ in the above algorithm is a relaxation parameter, and under-relaxation $\left(0<\omega^{k}<1\right)$ is considered here.

\subsection{Broyden method and Its Variation with Relaxation}

Broyden method $^{(17)}$ is a family of quasi-Newton method. Original Broyden method needs to memorize a $n \times n$ matrix through the calculation, where $n$ is the degree of freedom at the interface. Based on the point (3) described at the beginning of $\S 3$, we adopt the restart version instead of the original one. In the restart version, the calculation starts again using the solution obtained so far when iteration number reaches the user-defined parameter $t$.

Broyden method:

Step 0: Give an initial guess $\mathbf{d}_{\boldsymbol{\Gamma}}^{\mathbf{- 1}}$.

Step 1: Calculate $\mathbf{s}^{\mathbf{0}}=-\omega^{0}\left(\mathbf{d}_{\mathbf{\Gamma}}^{-\mathbf{1}}-S\left(F\left(M\left(\mathbf{d}_{\mathbf{\Gamma}}^{-\mathbf{1}}\right)\right)\right)\right)$.

Step 2: $\quad$ Set $\mathbf{d}_{\boldsymbol{\Gamma}}^{\mathbf{0}}=\mathbf{d}_{\boldsymbol{\Gamma}}^{-\mathbf{1}}+\mathbf{s}^{\mathbf{0}}, k=0$.

Step 3: Calculate $\mathbf{r}^{\mathbf{k}}=\mathbf{d}_{\Gamma}^{\mathbf{k}}-S\left(F\left(M\left(\mathbf{d}_{\Gamma}^{\mathbf{k}}\right)\right)\right)$.

Step 4: If converged, exit with the solution $\mathbf{d}_{\Gamma}^{\mathbf{k}}$. If not, go to Step 5.

Step 5: Calculate an increment $\mathbf{s}^{\mathbf{k}+\mathbf{1}}$ by the following process :

Step 5.0: $\quad$ set $\mathbf{p} \leftarrow-\mathbf{r}^{\mathbf{k}}$.

Step 5.1: for $i=0,1, \cdots, k-1$ :

$$
\begin{aligned}
& a=\omega^{i} / \omega^{i+1}, b=\omega^{i}-1 \\
& \mathbf{p} \leftarrow \mathbf{p}+\left(\mathbf{s}^{\mathbf{i}}, \mathbf{p}\right) /\left\|\mathbf{s}^{\mathbf{i}}\right\|^{2} \cdot\left(a \mathbf{s}^{\mathbf{i}+\mathbf{1}}+b \mathbf{s}^{\mathbf{i}}\right)
\end{aligned}
$$

Step 5.2: find an increment

$$
\mathbf{s}^{\mathbf{k}+\mathbf{1}} \leftarrow \omega^{k+1}\left(\mathbf{p}-\left(1-\omega^{k}\right) \mathbf{s}^{\mathbf{k}}\right) /\left(1-\omega^{k}\left(\mathbf{s}^{\mathbf{k}}, \mathbf{p}\right) /\left\|\mathbf{s}^{\mathbf{k}}\right\|^{2}\right)
$$

Step 6: $\quad$ Set $\mathbf{d}_{\Gamma}^{\mathbf{k}+\mathbf{1}}=\mathbf{d}_{\boldsymbol{\Gamma}}^{\mathbf{k}}+\mathbf{s}^{\mathbf{k}+\mathbf{1}}$.

Step 7: If $k=t$, restart. If not, set $k=k+1$ : go to Step 3.

$\omega_{k}$ in the above algorithm is a relaxation parameter, and here we consider under-relaxation $\left(0<\omega^{k}<1\right)$.

Hereafter, we will abbreviate the above-mentioned algorithms as follows: Block GaussSeidel method for GS, relaxed block Gauss-Seidel method by under-relaxation for GSUR, relaxed block Gauss-Seidel method by Aitken extrapolation for GSAT, Line Search method for LS, Jacobian-Free Newton-Krylov method for NK, relaxed Jacobian-Free Newton-Krylov method by under-relaxation for NKUR, Broyden method for BR and relaxed Broyden method by under-relaxation for BR. 


\section{Test Problems and Numerical Tests}

The following three model problems, which have features being representative of many other FSI problems with large deformation, are employed as test problems for the performance study on the eight nonlinear algorithms. The features appear especially in the parts of human body such as internal organs and blood vessel, and it has been pointed out that solving those problems by partitioned iterative method is challenging. As for control parameters peculiar to each method, $\omega_{k}$ for GSUR, $\omega_{0}$ for GSAT, $\beta$ for LS, $\epsilon_{0}$ for NK, $\epsilon_{0}, \omega_{k}$ for NKUR, $t$ for BR, and $t, \omega_{k}$ for BRUR are varied in the tests.

\subsection{Problem 1 : Collapsible Channel}

The first test problem is a steady flow in $2 \mathrm{D}$ collapsible channel ${ }^{(2)}$, as sketched in Fig. 1. In the collapsible channel, a part of one wall is a pre-stressed elastic wall, and then incompressible viscous fluid driven in the channel is influenced by the deformation of the elastic wall. The elastic wall is loaded by the external pressure $p_{\text {ext }}$ as well as the fluid force.

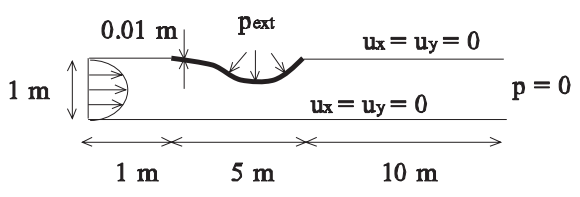

Fig. 1 Image of model problem.

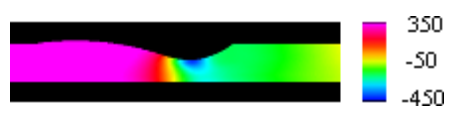

Fig. 2 Contour field for pressure at $\mathrm{Re}=500$.

For the fluid domain, inflow velocity with the average velocity $1.0 \mathrm{~m} / \mathrm{s}$ is given and viscosity is set as $\mu=1.0 \mathrm{~m}^{2} / \mathrm{s}$. Fluid density is controlled for changing Reynolds number Re. For the structure domain, Young's modulus of the elastic wall is set to be $E=10^{2} \mathrm{~N} / \mathrm{m}^{3}$. The elastic wall is subject to an initial stress $\sigma_{0}=10^{3} E$ which is assumed to be much larger than the additional stress generated by the wall deformation. The vertical wall displacement $d_{y}$ is measured at the control point which is $3.5 \mathrm{~m}$ from the left end of the wall. The finite element mesh has 1771 nodes and 3200 elements for the fluid part, and 102 nodes and 50 elements for the structure part.

In this parametric study, Reynolds number and the external pressure are controlled to change the degree of nonlinearity, which are known to affect the performance of each method in steady problems ${ }^{(2)}$. When Reynolds number becomes large, the FSI system develops largedisplacement of the elastic wall because of Bernoulli effect that causes pressure reduction in fluid in the most strongly collapsed part of the channel, as shown in Fig. 2.

In each analysis, convergence criterion is set so that 1 norm of $\mathbf{d}_{\mathbf{k}}-\mathbf{d}_{\mathbf{k}-\mathbf{1}}$ is less than $10^{-4}$ of its initial value (i.e. $\left\|\mathbf{d}_{\mathbf{k}}-\mathbf{d}_{\mathbf{k}-\mathbf{1}}\right\|_{1} \leq 10^{-4}\left\|\mathbf{d}_{\mathbf{1}}-\mathbf{d}_{\mathbf{0}}\right\|_{1}$ ), and the maximum iteration is also set to be 200. The selected values of control parameters are shown in the third and fourth row of Table 1. As for NKUR and BRUR, values for $\omega_{k}$ are shown in the third row, and ones for $\epsilon_{0}$ or $t$ are in the fourth row.

Table 1 shows the performance of each method for various combinations of Reynolds number and external pressure. The performance is evaluated by the number of fluid/structure solver calls because computational time varies depending on the initial values given for each solver. As for the number of solver calls of NK in Table 1, the one outside the parenthesis stands for the overall solver calls, while the one inside the parenthesis does the iteration number of Newton method itself. Furthermore, some methods converged to another solution in several cases, for which "-" is given in Table 1, due to the nonlinearity of the problem even though the calculation started from the initial values close enough to the equilibrium point. Those cases are excluded from the comparison.

At small Reynolds number of $\mathrm{Re}=100$, no significant difference is observed among the methods. NK and the methods with relaxation show rather bad performance requiring more solver calls than other methods. GS, GSAT and LS show almost the same performance, 
Table 1 Convergence property of each method ( $\infty$ : the method didn't converge until max iteration, $\times$ : the method stops due to bad quality of meshes or divergence of fluid/structure analysis, -: the method converges to another solution.)

\begin{tabular}{|c|c|c|c|c|c|c|c|c|c|c|c|c|c|c|c|}
\hline \multirow[t]{4}{*}{ Init. } & \multirow[t]{4}{*}{ Disp. $d_{y}$} & \multicolumn{14}{|c|}{ Its } \\
\hline & & \multirow{3}{*}{$\begin{array}{c}\text { GS } \\
-\end{array}$} & \multirow{3}{*}{$\begin{array}{c}\text { GSUR } \\
0.3\end{array}$} & \multicolumn{2}{|c|}{ GSAT } & \multicolumn{2}{|c|}{ LS } & \multirow{2}{*}{\multicolumn{2}{|c|}{$\begin{array}{c}\text { NK } \\
-\end{array}$}} & \multirow{2}{*}{\multicolumn{2}{|c|}{$\begin{array}{c}\text { NKUR } \\
0.5\end{array}$}} & \multirow{2}{*}{\multicolumn{2}{|c|}{$\begin{array}{c}\text { BR } \\
-\end{array}$}} & \multirow{2}{*}{\multicolumn{2}{|c|}{$\begin{array}{c}\text { BRUR } \\
0.5\end{array}$}} \\
\hline & & & & \multirow[t]{2}{*}{0.5} & \multirow[t]{2}{*}{0.1} & \multirow[t]{2}{*}{1.0} & \multirow[t]{2}{*}{0.6} & & & & & & & & \\
\hline & & & & & & & & \multicolumn{2}{|c|}{\begin{tabular}{l|l}
$10^{-1}$ & $10^{-2}$
\end{tabular}} & \multicolumn{2}{|c|}{\begin{tabular}{c|c}
\multicolumn{2}{c}{0.5} \\
$10^{-1}$ & $10^{-2}$
\end{tabular}} & \multicolumn{2}{|c|}{\begin{tabular}{l|l}
10 & 20
\end{tabular}} & \multicolumn{2}{|c|}{\begin{tabular}{l|l}
10 & 20
\end{tabular}} \\
\hline \multicolumn{16}{|c|}{$\mathrm{Re}=100$} \\
\hline-0.090 & -0.100 & 8 & 27 & 7 & 7 & 7 & 8 & $15(6)$ & $16(5)$ & $35(13)$ & 49(13) & 6 & 6 & 13 & 14 \\
\hline-0.190 & -0.200 & 9 & 28 & 9 & 9 & 8 & 10 & $13(5)$ & $18(5)$ & $37(13)$ & $53(14)$ & 7 & 7 & 14 & 14 \\
\hline-0.290 & -0.300 & 11 & 32 & 11 & 12 & 10 & 12 & $18(6)$ & $18(5)$ & $45(14)$ & $53(14)$ & 7 & 7 & 15 & 14 \\
\hline-0.390 & -0.400 & 16 & 33 & 16 & 17 & 11 & 14 & $20(6)$ & 19(5) & 51(14) & $56(14)$ & 8 & 8 & 15 & 14 \\
\hline \multicolumn{16}{|c|}{$\mathrm{Re}=250$} \\
\hline-0.090 & -0.100 & 25 & 79 & 13 & 12 & 14 & 21 & $23(7)$ & $23(6)$ & $54(15)$ & $70(15)$ & 9 & 9 & 17 & 16 \\
\hline-0.190 & -0.200 & 30 & 76 & 18 & 21 & 32 & 48 & $26(7)$ & $44(9)$ & $64(14)$ & $67(14)$ & 12 & 12 & 23 & 20 \\
\hline-0.290 & -0.300 & 68 & 140 & 131 & 127 & 27 & 49 & $30(7)$ & $28(6)$ & $65(14)$ & $90(16)$ & $x$ & $x$ & 32 & 23 \\
\hline-0.390 & -0.400 & $\infty$ & 175 & 177 & 186 & $\infty$ & 166 & $33(8)$ & $28(6)$ & $57(14)$ & $76(16)$ & 10 & 10 & 20 & 18 \\
\hline \multicolumn{16}{|c|}{$\mathrm{Re}=500$} \\
\hline-0.090 & -0.100 & - & - & 36 & 54 & 21 & 27 & $30(7)$ & $31(6)$ & $115(22)$ & $90(16)$ & 12 & 12 & 22 & 20 \\
\hline-0.190 & -0.200 & - & - & 156 & 149 & 29 & 35 & $37(8)$ & $46(8)$ & $88(18)$ & $100(17)$ & 24 & 20 & 24 & 23 \\
\hline-0.290 & -0.300 & $x$ & - & - & $\begin{array}{lll}- & & \end{array}$ & 43 & 73 & $x$ & $79(13)$ & $77(16)$ & $86(15)$ & $x$ & $x$ & 24 & 19 \\
\hline-0.390 & -0.400 & $x$ & $\infty$ & - & - & $x$ & 49 & $32(8)$ & $x$ & $76(18)$ & 198(39) & 16 & 13 & 23 & 18 \\
\hline
\end{tabular}

but only LS converges fast when the displacement becomes large. BR converges fast despite the amount of displacement. Increasing Reynolds number to $R e=250$ starts to cause a significant difference of performance among the algorithms. BR fails the calculation due to the divergence of fluid analysis at displacement $d_{y}=-0.3$, although it generally shows a fast convergence property. LS also shows good convergence property for $d_{y}=-0.1 \sim-0.3$, but only LS(0.6) succeeds to converge at $v=-0.4$. GSAT and NK type (i.e. NK and NKUR) converge stably in all the cases. However, efficiency of GSAT drastically deteriorates for large displacement cases, while that of NK type does not change much by the amount of deformation. At large Reynolds number of $\mathrm{Re}=500$, all the algorithms without relaxation fail to converge in more than one cases. Especially, GS lacks stability, showing many failure cases through the calculation. BR is again the fastest when converged, and LS converges with moderate speed in all cases.

In this test problem, GSAT, NK type, and BR type are slightly affected by the control parameters $\omega_{0}, \epsilon_{0}, t$ respectively. A small value for $\epsilon_{0}$ in NK improves efficiency of Newton iteration itself, but rather deteriorates entire efficiency, that is the number of solver calls in the Krylov method increases. Moreover, a large value for $t$ in BR improves efficiency as expected, but does not improve robustness. On the other hand, the relaxation parameters $\beta$ for LS, $\omega_{k}$ for NKUR and BRUR change efficiency and robustness of the algorithms. Setting small values for them improves the performance in stability while the speed is sacrificed.

The convergence histories for the cases of $\mathrm{Re}=250$ with displacement $d_{y}=-0.1,-0.3$ are plotted in Fig. 3. For the case of $d_{y}=-0.1$, GS needs more iterations to reach the
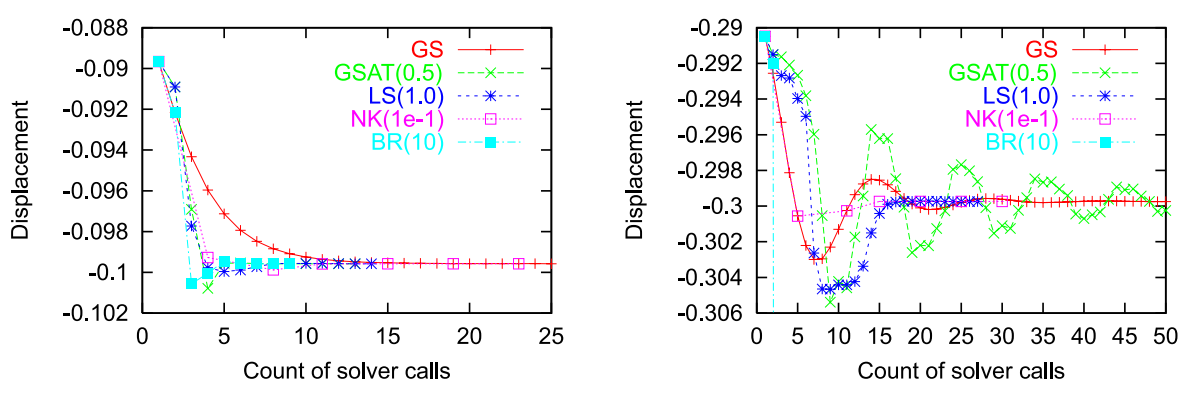

Fig. 3 Convergence history of wall displacement $\left(\operatorname{Re}=250\right.$, Left: $d_{y}=-0.100$, Right: $\left.d_{y}=-0.300\right)$. 
equilibrium point, even though excessive response is not observed. In other methods the excessive response occurs at the beginning of iterations. For the case of $d_{y}=-0.3$, the excessive response is observed in all the methods. BR results in a very large displacement of $d_{y}=-0.69$ at the third iteration, and then the calculation is stopped because of divergence of fluid analysis. GS and GSAT oscillates heavily after the excessive response, and requires lots of iterations to converge. On the other hand, LS reaches the equilibrium point smoothly after the excessive response. NK calculates an accurate displacement with extremely small amount of the excessive response in comparison with other methods.

Table 2 Dependency on Initial Values

\begin{tabular}{|c|c|c|c|c|c|c|c|c|c|}
\hline \multirow[t]{4}{*}{ Init. } & \multirow[t]{4}{*}{ Disp. $d_{y}$} & \multicolumn{8}{|c|}{ Its } \\
\hline & & GS & GSUR & GSAT & LS & NK & NKUR & $\mathrm{BR}$ & BRUR \\
\hline & & \multirow[t]{2}{*}{-} & \multirow[t]{2}{*}{0.3} & \multirow[t]{2}{*}{0.5} & \multirow[t]{2}{*}{1.0} & - & 0.5 & - & 0.5 \\
\hline & & & & & & $10^{-1}$ & $10^{-1}$ & 10 & 10 \\
\hline \multicolumn{10}{|c|}{$\Delta d_{y}=0.030$} \\
\hline-0.070 & -0.100 & 25 & 78 & 10 & 14 & $x$ & $53(17)$ & 8 & 17 \\
\hline-0.170 & -0.200 & 30 & 78 & 19 & 27 & $30(8)$ & $70(20)$ & 12 & 23 \\
\hline-0.270 & -0.300 & 68 & 141 & 96 & 31 & $61(14)$ & $74(17)$ & $x$ & $x$ \\
\hline-0.370 & -0.400 & $\infty$ & 176 & 178 & $\infty$ & $x$ & $\times$ & 11 & 22 \\
\hline \multicolumn{10}{|c|}{$\Delta d_{y}=0.050$} \\
\hline-0.050 & -0.100 & 25 & 77 & 10 & 14 & $x$ & $125(35)$ & 8 & 18 \\
\hline-0.150 & -0.200 & 30 & 80 & 17 & 26 & $41(10)$ & $x$ & 10 & 23 \\
\hline-0.250 & -0.300 & 69 & 142 & 121 & 33 & $\times$ & $73(17)$ & $x$ & $x$ \\
\hline-0.350 & -0.400 & $\infty$ & 177 & 180 & 156 & $x$ & $\times$ & $x$ & 24 \\
\hline
\end{tabular}

Table 2 shows performances of the eight methods when changing the initial values. $\Delta d_{y}$ in this table indicates the distance between initial and final wall displacement in vertical direction. GS type and LS converge with almost the same iteration number despite the initial values. On the other hand, NK type and BR type show remarkable deterioration when the initial values are set far from the final points. Those methods tend to develop excessive deformation and their solutions oscillate seriously when starting from the point far from the equilibrium one, as shown in Fig. 4. Finally, the calculation is stopped due to bad mesh quality or the divergence of fluid/structure analysis.
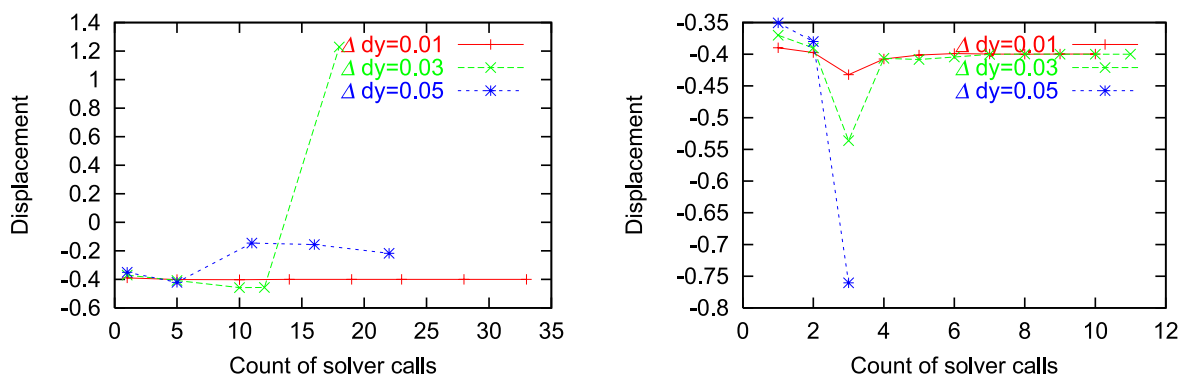

Fig. 4 Convergence history of wall displacement $\left(\operatorname{Re}=250, d_{y}=0.4\right.$, Left: NK, Right: BR).

\subsection{Problem 2 : Cavity with Flexible Bottom Membrane}

The second problem is an unsteady flow in 2D cavity with flexible bottom membrane ${ }^{(11)}$, as sketched in Fig. 5. This problem is a modification of the well known wall-driven cavity flow problem often used as benchmark in fluid dynamics. The original constant velocity at the top is changed for a periodical velocity $\bar{u}=1-\cos \left(\frac{2 \pi t}{5}\right)$. At the upper part of right side, there are unconstrained nodes that allow free outflow of fluid, so that the structural displacements are not constraint by fluid's incompressibility.

The fluid properties assumed are a density $\rho^{F}=1.0 \mathrm{~kg} / \mathrm{m}^{3}$ and a viscosity $v=0.01 \mathrm{~m}^{2} / \mathrm{s}$. For the structure, Young's modulus is set to be $E=250.0 \mathrm{~N} / \mathrm{m}^{3}$ and a Poisson's ratio $v=0.0$. The mesh of the fluid domain consists of 1,089 nodes and 2,048 elements, and that of the structure domain does 99 nodes and 64 elements. 


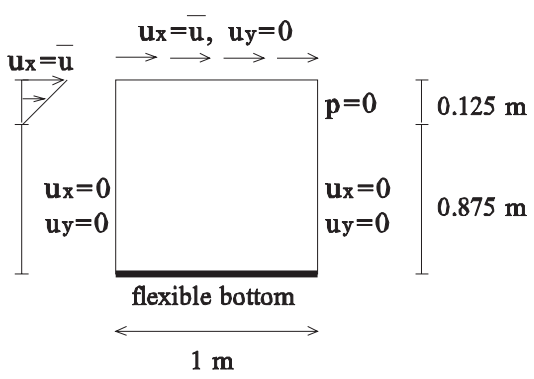

Fig. 5 Image of model problem.

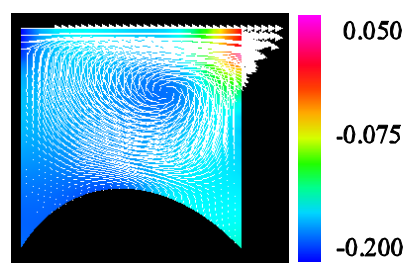

Fig. 6 Contour field for pressure and arrow for velocity $|\mathbf{v}|$ at $t=19.5 \mathrm{~s}$

In this problem, the calculation is carried out by changing the mass density ratio of fluid and structure density $\rho^{F} / \rho^{S}$ and the time step $\Delta t$, which are known to have a great influence on the performance of each method in unsteady problems ${ }^{(8)}$. Changing the mass density ratio of fluid and structure results in the change of added mass effect.

Each calculation is carried out from $t=0$ to $50 \mathrm{~s}$, and the average solver calls per time step are given in Table 3. The convergence criteria is set as $\left\|\mathbf{d}_{\mathbf{k}}-\mathbf{d}_{\mathbf{k}-\mathbf{1}}\right\|_{1} \leq 10^{-3}\left\|\mathbf{d}_{\mathbf{1}}-\mathbf{d}_{\mathbf{0}}\right\|_{1}$, and the maximum iteration is 200 in each time step. The control parameters are set as shown in the third row of Table 3.

Table 3 Convergence property of each method

\begin{tabular}{|c|c|c|c|c|c|c|c|c|c|c|c|c|c|c|}
\hline \multirow[t]{4}{*}{$\rho^{F} / \rho^{S}$} & \multirow[t]{4}{*}{$\Delta t$} & \multicolumn{13}{|c|}{ Its } \\
\hline & & \multirow[t]{3}{*}{ GS } & \multicolumn{2}{|c|}{ GSUR } & \multicolumn{2}{|c|}{ GSAT } & \multicolumn{2}{|c|}{ LS } & \multirow{2}{*}{\multicolumn{2}{|c|}{$\begin{array}{c}\text { NK } \\
-\end{array}$}} & \multirow{2}{*}{\multicolumn{2}{|c|}{$\begin{array}{c}\text { NKUR } \\
0.6\end{array}$}} & \multirow{3}{*}{$\begin{array}{c}\text { BR } \\
- \\
10\end{array}$} & \multirow{3}{*}{$\begin{array}{c}\text { BRUR } \\
0.6 \\
10\end{array}$} \\
\hline & & & \multirow[t]{2}{*}{0.3} & \multirow[t]{2}{*}{0.1} & \multirow[t]{2}{*}{0.6} & \multirow[t]{2}{*}{0.3} & \multirow[t]{2}{*}{0.6} & \multirow[t]{2}{*}{0.3} & & & & & & \\
\hline & & & & & & & & & $10^{-1}$ & $10^{-2}$ & $10^{-1}$ & $10^{-2}$ & & \\
\hline \multicolumn{15}{|c|}{ dependency on $\rho^{S}$} \\
\hline 0.002 & 0.1 & $x$ & 21.0 & 66.9 & 5.3 & 5.6 & 9.7 & 14.7 & $\infty$ & $7.4(2.1)$ & $17.5(8.0)$ & $23.7(8.1)$ & 3.9 & 9.3 \\
\hline 0.010 & 0.1 & $x$ & $\times$ & 67.0 & 9.9 & 10.4 & 12.1 & 18.2 & $\infty$ & 14.1(3.5) & $\infty$ & $30.4(8.7)$ & 6.2 & $x$ \\
\hline 0.017 & 0.1 & $x$ & $x$ & 67.0 & 17.4 & 16.9 & 15.0 & 19.2 & $\infty$ & $17.7(4.1)$ & $\infty$ & $35.9(9.0)$ & $x$ & $x$ \\
\hline 0.033 & 0.1 & $x$ & $x$ & 67.1 & $\infty$ & $\infty$ & $\infty$ & 22.6 & $\infty$ & $\infty$ & $\infty$ & $\infty$ & $x$ & $x$ \\
\hline \multicolumn{15}{|c|}{ dependency on $\Delta t$} \\
\hline 0.002 & 0.1 & $x$ & 21.0 & 66.9 & 5.3 & 5.6 & 9.7 & 14.7 & $\infty$ & $7.4(2.1)$ & $17.5(8.0)$ & $23.7(8.1)$ & 3.9 & 9.3 \\
\hline 0.002 & 0.05 & $x$ & 21.0 & 67.0 & 4.9 & 5.3 & 9.6 & 14.7 & $\infty$ & $6.5(2.0)$ & $17.2(8.0)$ & $19.8(8.2)$ & 3.9 & 9.3 \\
\hline 0.002 & 0.01 & $x$ & 21.0 & 67.0 & 4.9 & 4.8 & 10.9 & 10.5 & $\infty$ & $6.2(1.8)$ & $\infty$ & $24.8(8.4)$ & 3.9 & 10.9 \\
\hline
\end{tabular}

As can be seen in Table 3, increasing the mass density ratio results in increasing the number of solver calls for most of the methods. Especially, the solver call increase is remarkable for GSAT and NK type, since GSAT(0.6), GSAT(0.3), NK(10 $\left.{ }^{-2}\right)$, and $\operatorname{NKUR}\left(0.6,10^{-2}\right)$ need more than the max iterations at $t=2.6,18.0,19.3,29.4$, respectively for the largest mass density ratio $\rho^{F} / \rho^{S}=0.033$. LS also gets worse as the mass density ratio increases, but with relaxation it stably converges with a small iteration number through the calculation for $\rho^{F} / \rho^{S}=0.033$. GS diverges in all the cases. Under-relaxation technique improves its stability, but they spend much more iterations than other methods. BR converges very fast for small mass density ratio, but it becomes unstable for large mass density ratio and stops the calculation due to the bad mesh quality or the divergence of structure analysis.

Figure 7 shows the convergence property of each method for the cases of $\rho^{F} / \rho^{S}=0.002$ with $\Delta t=0.1,0.01$. Although the dependency on the time step is not so clear compared to that on the mass density ratio from Table 3, Fig. 7 indicates more iterations are required at some time steps when the time increment decreases to $\Delta t=0.01$. In particular, the increase of the solver calls for NK is conspicuous.

In this problem, all the methods except GSAT are more affected by the control parameters. The parameter $\beta$ for LS and $\omega_{k}$ for GSUR, NKUR have relaxation characteristics, so selecting small values attains stability for large mass density ratio. On the other hand, selecting small values for $\omega_{k}$ in BRUR rather amplifies instability for large mass density ratio, as a solution suddenly goes far from the equilibrium point during the iteration. Furthermore, 

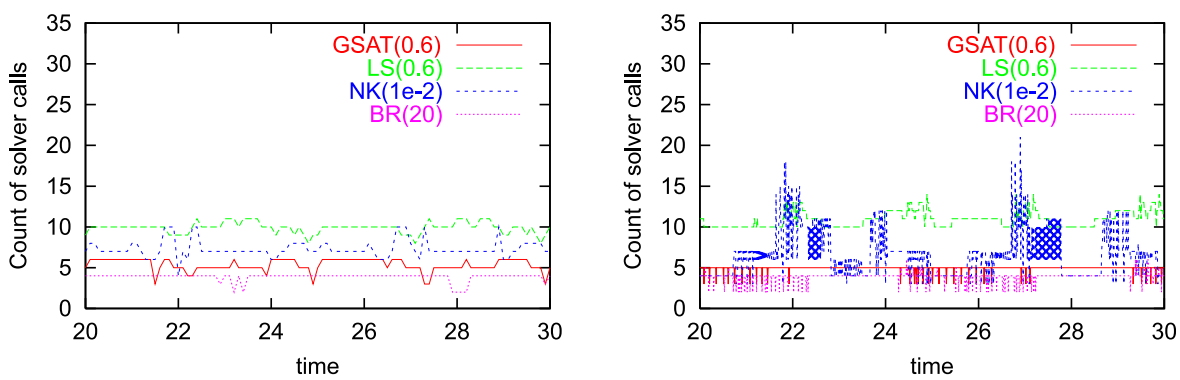

Fig. 7 Count of solver calls $\left(\rho^{F} / \rho_{S}=0.002\right.$, Left: $\Delta t=0.1$, Right: $\left.\Delta t=0.01\right)$.

the tolerance for Krylov Subspace method $\epsilon_{0}$ for NK type takes an important part since the displacement increment becomes less accurate with a larger tolerance value and NK type take many iteration counts after all. The performance of GSAT is not influenced by the parameter $\omega_{0}$, irrespective of the amounts of structural density and time step.

\subsection{Problem 3 : Channel with Flexible Wall}

The third problem is an unsteady flow in 2D channel with flexible wall, as sketched in Fig. 8. This problem was originally proposed by Mok and Wall ${ }^{(10)}$ and $\mathrm{Mok}^{(11)}$.

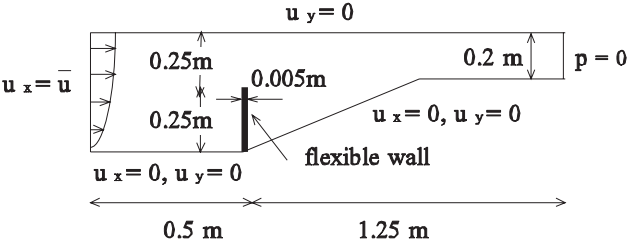

Fig. 8 Image of model problem.

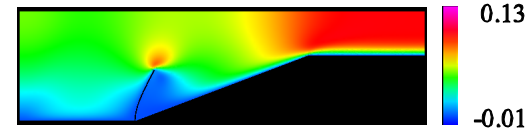

Fig. 9 Contour field for $\mathrm{x}$-velocity component at $t=7.5 \mathrm{~s}$.

The material properties of the structure part are Young's modulus $E=2.3 \times 10^{6} \mathrm{~N} / \mathrm{m}^{2}$ and Poisson's ratio $v=0.45$. Those of the fluid part are the mass density $\rho^{F}=956.0 \mathrm{~kg} / \mathrm{m}^{3}$ and the viscosity $\mu=0.145$. The inflow velocity is parabolic and applied gradually increasing up to $10 \mathrm{~s}$.

$$
\bar{u}=\frac{0.06067}{2}\left(1-\cos \frac{\pi t}{10}\right)(t<10), \quad \bar{u}=0.06067(t \geq 10) .
$$

The finite element meshes have 8,200 nodes and 15,801 elements for the fluid part, and 405 nodes and 320 elements for the structure part.

In this problem, the mass density ratio of fluid and structure $\rho^{F} / \rho^{S}$ and time step $\Delta t$ are changed as in the problem 2. Each calculation is performed between $t=0 \sim 20 \mathrm{~s}$, and the average solver calls per time step are shown in Table 4 . The same conditions as the last cavity problem are set for convergence criteria and the maximum iteration number.

Table 4 shows similar dependency of convergence property on the mass density ratio to

Table 4 Convergence property of each method

\begin{tabular}{|c|c|c|c|c|c|c|c|c|c|c|c|}
\hline \multirow[t]{4}{*}{$\overline{\rho^{F} / \rho^{S}}$} & \multirow[t]{4}{*}{$\Delta t$} & \multicolumn{10}{|c|}{ Its } \\
\hline & & \multirow{3}{*}{$\begin{array}{c}\text { GS } \\
-\end{array}$} & \multirow{3}{*}{$\begin{array}{c}\text { GSUR } \\
0.1\end{array}$} & \multicolumn{2}{|c|}{ GSAT } & \multicolumn{2}{|c|}{ LS } & \multirow{2}{*}{$\begin{array}{c}\text { NK } \\
-\end{array}$} & \multirow{2}{*}{$\frac{\mathrm{BR}}{-}$} & \multicolumn{2}{|c|}{ BRUR } \\
\hline & & & & \multirow[t]{2}{*}{0.5} & \multirow[t]{2}{*}{0.1} & \multirow[t]{2}{*}{0.5} & \multirow[t]{2}{*}{0.1} & & & 0.8 & 0.4 \\
\hline & & & & & & & & $10^{-1}$ & 20 & 20 & 20 \\
\hline \multicolumn{12}{|c|}{ dependency on $\rho^{S}$} \\
\hline 0.637 & 0.1 & $x$ & $x$ & 18.7 & 40.0 & 19.8 & 22.1 & $\infty$ & 7.5 & 9.8 & $x$ \\
\hline 0.956 & 0.1 & $x$ & 65.8 & $\infty$ & $\infty$ & 22.3 & 28.4 & $\infty$ & 8.2 & 10.1 & $x$ \\
\hline 1.912 & 0.1 & $x$ & $\times$ & $\infty$ & $\infty$ & 40.0 & 51.3 & $\infty$ & $\times$ & $x$ & $x$ \\
\hline \multicolumn{12}{|c|}{ dependency on $\Delta t$} \\
\hline 0.637 & 0.2 & $x$ & 65.0 & $\infty$ & $\infty$ & 13.5 & 23.7 & $\infty$ & $x$ & 9.4 & $x$ \\
\hline 0.637 & 0.1 & $x$ & $x$ & 18.7 & 39.2 & 19.8 & 22.8 & $\infty$ & 7.8 & 9.8 & $x$ \\
\hline 0.637 & 0.05 & $x$ & 66.0 & 21.7 & 35.5 & 36.5 & 30.6 & $\infty$ & 8.2 & 10.1 & $x$ \\
\hline
\end{tabular}


the previous problem 2. GS diverges for even the smallest mass density ratio $\rho^{F} / \rho^{S}=0.637$, and GSUR(0.1) also fails in some cases. GSAT and LS converge in reasonable speed for $\rho^{F} / \rho^{S}=0.637$, but only LS converges stably for larger mass density ratio. NK $\left(10^{-1}\right)$ does not converge within 200 iterations in the Krylov solver even at the first iteration of the first time step. BR shows the fastest characteristics in small mass density ratio, but it becomes unstable for large mass density. Especially, BRUR deteriorates its stability, i.e. BR(0.4) fails in all the cases during the calculation, as is the case of problem 2.

The dependency of performance on time step is rather clear in this problem. The performance of GSAT becomes extremely worse as time step increases. GSAT( 0.5$)$ fails to converge until the max iteration at $t=4.3$, and $\operatorname{GSAT}(0.1)$ does at $t=5.2$ with $\Delta t=0.2$. BR shows an instable property for larger time step, i.e. it fails the calculation at $t=7.6$ for $\Delta t=0.2$. BRUR converges stably in that case. Such a feature of BR type in this problem is basically similar to the feature observed in problem 1 when changing the initial values. $\operatorname{LS}(0.5)$ and $\operatorname{LS}(0.1)$ again succeed to converge in all cases, although their efficiency deteriorates as time step decreases.

\section{Conclusion}

The selected four nonlinear algorithms, i.e. Block Gauss-Seidel method (GS), Line Search method (LS), Jacobian-Free Newton-Krylov method (NK), and Broyden method (BR), and their relaxed variations, i.e. relaxed block Gauss-Seidel method by under-relaxation (GSUR), relaxed block Gauss-Seidel method by Aitken extrapolation (GSAT), relaxed Jacobian-Free Newton-Krylov method by under-relaxation (NKUR), and relaxed Broyden method by under-relaxation (BRUR) have been applied to solve the partitioned iterative FSI problems, taking three kinds of test problems, i.e. the steady flow in 2D collapsible channel, the unsteady flow in 2D cavity with flexible bottom membrane, and the unsteady flow in 2D channel with flexible wall. Practical performances of the algorithms have been investigated in detail through intensive parametric studies. Especially, we have examined the influence of Reynolds number Re, amount of deformation $d_{y}$, and initial values for the steady problem, and those of the mass density ratio of fluid and structure $\rho^{F} / \rho^{S}$ and time step $\Delta t$ for the unsteady problems, and those of control parameters peculiar to each method for both steady and unsteady problems. Since it was difficult to discuss the performances quantitatively, we carried out qualitative discussion. The main features of the algorithms for solving the partitioned iterative FSI problems can be summarized as follows:

- GS shows slow convergence even in steady problems with small Re and $d_{y}$, while its performance hardly gets affected by initial values. It also lacks stability in steady problems with large Re and $d_{y}$, and in unsteady problems despite $\rho^{F} / \rho^{S}$ and $\Delta t$. GSUR improves stability, and it successfully converges according to a parameter even in the problems where GS fails to converge. However, in all the cases, its performance in efficiency is very poor. GSAT basically shows good performance among the algorithm. It converges fast in problems with small $\operatorname{Re}, d_{y}, \rho^{F} / \rho^{S}$, and $\Delta t$. However, its performance in efficiency and robustness notably deteriorates despite control parameters when above physical parameters get large.

- LS shows good performance among the algorithm. LS shows moderate performance in efficiency for all the tested cases, while it is not the fastest compared to other algorithms. It also shows good performance in robustness within the moderate speed even in the problems with large Re, $d_{y}$, and $\rho^{F} / \rho^{S}$ when selecting an appropriate control parameter.

- In most cases, NK shows poor efficiency and lack of robustness. The performance of NK strongly depends on control parameters, and it does not work for unsteady problems, irrespective of selection of the parameters. NKUR improves stability compared to NK, but its efficiency is poor as is the case of GSUR.

- BR shows the best performance in efficiency in problems with small Re, $d_{y}$, and $\rho^{F} / \rho^{S}$ among all the algorithms. It, however, lacks stability when those physical parameters get large. Furthermore, it sometimes fails to converge as initial values are set far from the final values, or a large $\Delta t$ is set. BRUR improves stability in problems with large Re, $d_{y}$, and $\Delta t$, while its 
speed deteriorates compared to BR. On the other hand, it rather amplifies instability as $\rho^{F} / \rho^{S}$ becomes large.

The above knowledge will be useful in selecting appropriate nonlinear algorithms and their control parameters for partitioned iterative FSI problems, and in developing more efficient and robust nonlinear algorithms suitable for a general purpose large-scale parallel FSI analysis system.

\section{References}

( 1 ) Q. Zhang and T. Hisada, "Investigations of the Coupling Methods for FSI Analysis by FEM", Trans. J. Soc. Mech. Eng. A, Vol. 67-662, pp. 1-8, 2001.

( 2 ) M. Heil, "An efficient solver for the fully-coupled solution of large-displacement fluidstructure interaction problems", Com. Meth. App. Mech. Eng., Vol. 193, pp. 1-23, 2004.

( 3 ) S. Yoshimura, M. Okamoto, and T. Yamada, "Stability and Efficiency of an Iterative Partitioned Coupling Algorithm for Fluid-Structure Interaction Problems", Trans. J. Soc. Mech. Eng. B, Vol. 72-716, pp. 17-24, 2006.

( 4 ) T. Yamada, T. Otsuka, and Y. Ito, "Numerical Simulation of Interaction of Membrane and Fluid by an Iterative Coupling Algorithm", Proc. Conf. C.E.S., Vol. 10, pp. 21-24, 2005.

( 5 ) T. Yamada and S. Yoshimura, "Line Search Partitioned Approach for Fluid-structure Interaction Analysis of Flapping Wing", Comp. Model. Eng. Sci., Vol. 24-1, pp. 51-60, 2008.

( 6 ) J. F. Gerbeau and M. Vidrascu, "A quasi-Newton algorithm based on a reduced model for fluid-structure interaction problems in blood flows", Math. Model. Num. Anal., Vol. 37(4), pp. 631-647, 2003.

( 7 ) H. G. Matthies, R. Niekamp, J. Steindorf, "Algorithms for strong coupling procedures", Comp. Meth. App. Mech. Eng., Vol. 195, pp. 2028-2049, 2006.

( 8 ) U. Kuttler, and W. A. Wall, "Fixed-point fluid-structure interaction solvers with dynamic relaxation", Comp. Mech., Vol. 43, pp. 61-72, 2008.

( 9 ) S. Deparis, "Numerical analysis of axisymmetric flows and methods for fluid-structure interaction arising in blood flow simulation", $\mathrm{PhD}$ thesis, Ecole Polytechnique Federale de Lausanne, 2004.

(10) D. P. Mok and W. A. Wall, "Partitioned analysis schemes for the transient interaction of incompressible flows and nonlinear flexible structures", Trends Comp. Str. Mech., Barcelona, CIMNE, 2001, pp. 689-698.

(11) D. P. Mok, "Partitionierte Lösungsansätze in der Strukturdynamik und der FluidStruktur - Interaktion", PhD thesis, Institut fur Bautstatik, Universitat Stuttgart, 2001.

(12) S. Yoshimura, R. Shioya, H. Noguchi and T. Miyamura, "Advanced general-purpose computational mechanics system for large-scale analysis and design", J. Comp. App. Math., Vol. 49, 2002, pp. 279-296.

(13) Development of Computational Mechanics System for Large Scale Analysis and Design (ADVENTURE Project), http://adventure.q.t.u-tokyo.ac.jp/

(14) The project of "Revolutionary Simulation Software", http://www.ciss.iis.u-tokyo.ac.jp/ rss21/

(15) K. Stein, T. E. Tezduyar, and R. Benny, "Automatic mesh update with the solidextension mesh moving technique", Comp. Meth. App. Mech. Eng., Vol. 193, 2004, pp. 2019-2032.

(16) P. George, F. Hecht, and E. Saltel, "Automatic mesh generation with specified boundary", Comp. Meth. App. Mech. Eng., Vol. 92, 1991, pp. 269-288.

(17) C. T. Kelley, "Solving Nonlinear Equations with Newton's Method", SIAM, 2003.

(18) T. Kolda and R. Pawlowski et al. NOX: An object-oriented nonlinear solver package. http://software.sandia.gov/trilinos/packages/nox, 2005. 